\title{
XV. The recent eclipse results and Stokes-Plack's æther
}

\section{Silberstein Ph.D.}

To cite this article: L. Silberstein Ph.D. (1920) XV. The recent eclipse results and Stokes-Plack's æther, Philosophical Magazine Series 6, 39:230, 161-170, DOI: $10.1080 / 14786440208636027$

To link to this article: http://dx.doi.org/10.1080/14786440208636027

曲 Published online: 17 May 2010.

Submit your article to this journal ¿

山 Article views: 10

Q View related articles $\square$ 
THE

LONDON, KDINBURGH, AND DUBLIN

\title{
PHILOSOPHICAL MAGAZINE
}

\author{
AND \\ JOURNAL OF SCIENCE.
}

[SIXTH SERIE8.]

FEBRUARY 1920.

XV. The recent Eclipse Results and Stokes-Planck's AEther. By L. Silberstein, Ph.D., Lecturer in Mathem. Physics at the University of Rome*.

1. T $T$ is well known that, in 1845, Stokes proposed a 1 theory of aberration (Phil. Mag. xxvii. p. 9), which was based on the assumption that the luminiferous æether surrounding our planet is dragged along in its annual motion so that the velocity of the ether relative to the Earth is nil at its surface, and, increasing continuously, becomes equal and opposite to the Earth's velocity at very large distances from the Earth or, to put it short, at infinity. The purpose of this hypothesis, as opposed to that of Fresnel's stagnant æther, was to give a rigorous independence of all purely terrestrial optical experiments from the Earth's annual motion (combined with that of the solar system). In order to account for the semi-terrestrial phenomenon known as astronomical aberration, Stokes had to assume that the motion of the æther, between the Earth and the stars in question, is purely irrotational. But, by a well-known theorem of hydrodynamics, this assumption was not compatible with the incompressibility of Stokes's æether and, at the same time, with the absence of slipping over the Earth's surface.

2. In order to overcome this essential difficulty Max Planck has suggested that the incompressibility could be

$$
\text { * Communicated by Sir Oliver Lodge. }
$$

Phil. Mag. S. 6. Vol. 39. No. 230. Feb. 1920. 
given up * and replaced by the assumption that the other is condensed round the Earth, and other celestial bodies, as if it were subjected to the force of gravitation and behaved more or less like a perfect gas. Lorentz, in spite of his personal preference for a fixed æther, took up Planck's idea and worked out the problem under the special (but by no means the only possible) assumption that the æether density $\rho$ and pressure $p$ obey Boyle's law, $\rho=\alpha p$, where $\alpha=$ const. If $M$ lo the Earth's mass, in astronomical units, this gives

$$
\rho=\rho_{\infty} e^{a M / r}, . \quad . \quad . \quad . \quad . \quad .
$$

where $\rho_{\infty}$ is the density at infinity and $r$ the distance of any external point from the Earth's centre. The maximum velocity of slip at the Earth's surface $(r=R)$, in the direction opposite to that of its motion becomes $\uparrow$

$$
v={ }_{4}{ }_{4} \cdot \frac{\sigma^{3}}{e^{\sigma}-\left(1+\sigma+\frac{1}{2} \sigma^{2}\right)}, \quad . \quad . \quad .
$$

where $\sigma=\alpha M / R$, and $v_{\infty}$ is the velocity of the rether, relative to the Earth, at infinity.

To account for the astronomical aberration within the limits of experimental error it is necessary and sufficient to make $v=\frac{1}{100} v_{\infty}$. This gives, by (2), with sufficient approximation (since the required $\sigma$ is manifestly so large as to make the second term of the denominator negligible),

$$
\sigma^{3} \doteqdot 0 \cdot 04 e^{\sigma}
$$

so that the said requirement is amply satisfied by

$$
\sigma=10 \cdot 2 \text {. . . . . . . }
$$

This means, according to (1), a condensation $\ddagger$ of the xther amounting at the Earth's surface to little less than

$$
s \equiv \frac{\rho}{\rho_{\infty}}=27000,
$$

and gives at the same time for the (lower limit of the) coefficient $\alpha$ the value $10 \cdot 2 R / M$, to which we may return

* $\quad$ 'f. H. A. Lorentz's paper on Stokes's theory of aberration in Amsterdam Proc. for 1898-99, p. 443, reprinted in vol. i. of his Abhandlungen.

$\dagger$ A short deduction of this formula will be found in Lorentz's 'Theory of Electrons,' 1909, p. 314.

‡ What is commonly called "condensation." would in our case be $\frac{\rho}{\rho_{\infty}}-1$. But it will be convenient to use this as a short name for $\rho / \rho_{\infty}$, which will henceforth be denoted by $s$. 
later on. In order to reduce the slip to $\frac{1}{2}$ per cent. of $v_{\infty}$ a condensation of about 60000 would be required *

In view of this considerable condensation, required by the theory of Stokes-Planck, Lorentz made in 1909 ('Theory of Electrons,' pp. 173-4) the following characteristic remark:-

"In this department of physics, in which we can make no progress without some hypothesis that looks somewhat startling at first sight, we must be careful not rashly to reject a new idea, and in making his suggestion Planck has certainly done a good thing. Yet I dare say that this assumption of an enormously condensed ether, comlined, as it must be, with the hypothesis that the velocity of light is not in the least altered by it, is not very satisfactory." [The last words are italicised for our present purpose.

In fact, such a condensation, introduced $a \bar{d}$ hoc and serving only the negative purpose of not upsetting the theory of aberration, did not seem very satisfactory, and the present writer has as recently as 1914 expressed the same opinion in his book on Relativity (p. 63), not so much to defend Fresnel's and Lorentz's fixed æther, as to prepare the reader's mind for the complete abolition of the rether and thus to introduce him to Einstein's "special" relativity of 1905. Such has been the position of things until recently.

3. Now, it so happens that, stimulated by the desire to test Einstein's generalized relativity and theory of gravitation, the astronomers participating in the last Eclipse Expedition have found an undoubtedly positive effect, the bending of rays passing near the Sun. As I have pointed ont on previous occasions, it seems premature to interpret this result as a verification of Einstein's theory, not merely in view of the small outstanding discrepancies, but chiefly in view of the failure of detecting the spectrum shift predicted by the theory, with which the whole theory stands or falls. But the Eclipse result proves at any rate that there is an "alteration," a change of light-velocity all around the Sun, which thus invalidates the words of Lorentz italicized in the quotation above. The condensation claimed by Planck's modification of Stokes's theory, for the Sun as well as for the Earth and for all other material bodies, is no longer devoid of influence on observable phenomena. It suddenly acquires physical life, so to speak.

* Notice that the aberration is a first order effect, while such phenomena as that expected by Michelson-Morley are second order effects $\left(v^{2} / c^{2}\right)$, so that the above condensation suiting the aberration up to 1 per cent. will reduce the Michelson-Morley effect to one tenthousandth of its value, and thus practically annihilate it. There is thus no need for making $\sigma$ larger than $10 \cdot 2$. 


\section{Dr. I. Silberstein on the recent Eclipse Results}

In other words, the discovery made at Brazil naturally suggests the idea that the observed deflexion is due to the condensation of the cether around the Sun *, and although one has been an implacable enemy of any æther at all, for the last fifteen years, one does not hesitate to point out this possibility - a last glimpse of hope, perhaps, for the banished medium.

Let us imagine for the moment that Einstein had never published his debatable, though undoubtedly beautiful, new theory-not even that of 1905 . Then it is almost certain that the Eclipse result would readily be acclaimed as an evidence of the condensation of the zther near the Sun, as required by the theory of Stokes-Planck, and would encourage the physicists to work out in detail the optical and associated consequences of such a condensation. But even though Einstein's theory has been published, and is being made popular in a most sensational way, we cannot help elinging to the said idea. I just learn from "The Observatory" for August that Mr. Jonckheere suggested some months ago that refractions may, inter alias, be caused by "a hypothetical condensation of ether near the Sun." My point, however, is that such a source of. refraction acquires a particular interest if it is treated in connexion with the half-forgotten theory of Stokes-Planck, when it eeases to be a detached hypothesis.

It is in this sense and in such an organic connexion that I should like to draw attention to this aspect of the subject.

Of course, the quantitative details of the suitable modification of the optical, or the electromagnetic, properties of the wether due to a radially symmetrical or any other condensation have to be worked out carefully. It is not the purpose of this Note to give a complete investigation of this kind, but only some bints at its possibility. Such hints, together with some remarks on the possible advantages of the adrocated theory, will occupy our attention in the following sections.

4. If, merely to fix the ideas, the Boyle law is still adhered to, the condensation $s=\rho / \rho_{\infty}$ outside a radially symmetrical gravitating mass is given, as in (1), by

$$
\log s=\frac{\alpha M}{r} \text {. . . . . . . }
$$

If we assume, for places near the Earth's surface, not more and not less than what is just needed for the theory of

* The logarithm of this condensation would amount, at' the Sun's surface, by (1) and (E), to the evormous tigure $\sigma=\log s \doteqdot 31100$. ('f. the following footnote. 
aberration, i. e. $\sigma=\log s=10^{\circ} 2$, we shall have at the surface of the Sun, as already mentioned in a footnote,

$$
\sigma=\log s=10 \cdot 2 \frac{12 \cdot 8}{42} 10^{4} \doteqdot 31000, . . .
$$

which means, no donbt, an enormous condensation*. The corresponding relative velocity of slipping $v / v_{\infty}$ will, by (2), be almost evinescent; the drag will be almost complete.

On the other hand, at the surface of a hydrogen atom, assumed for the moment to be a homogeneous sphere (and the only existing body), we shall have $\log s=1 \cdot 7 \cdot 10^{-34}$, that is to say,

$$
s=\frac{\rho}{\rho_{\infty}} \doteqdot 1+1 \cdot 7.10^{-34}
$$

indistinguishable from unity. Notice that for small $\sigma$ the denominator in (2) reduces" to ${ }_{6}^{3} \sigma^{3}+{ }_{2}^{3} \sigma^{4}+$ higher terms, so that the relative slip becomes

$$
\frac{v}{i_{0}} \div \frac{3}{2}\left(1-\frac{1}{4} \sigma\right) \text {. . . . . . . . . }
$$

For such bodies, therefore, as a hydrogen atom, or in fact any other atom, the ratio in question will be exceedingly nearly equal its limiting value $3 / 2$, which is well known to be the maximum relative slipping for a sphere moving in an incompressible liquid. In short, for such small bodies there will be practically no drag at all. The more so for electrons, if one wished to attribute to them gravitational properties. This behaviour will be important in connexion with some such electrodynamic theories of ponderable media, as is that proposed by Lorentz, which require a complete slip. But even a sphere of the mass of $1 \mathrm{~kg}$. and the radius of $10 \mathrm{~cm}$., for which $\sigma=1 \cdot 09 \cdot 10^{-16}$, will practically have no " grip upon the æther." This will readily be seen to account, among other things, for the negative results of Sir Oliver Lodge's ingenious experiments with the Ether machine, even if its whirling part were made much more massive. As a mere curiosity notice that even the Moon would have only a partial, weak grip upon our rehabilitated æether. In fact, at the Moon's surface we should have $\sigma=10.2 \times 0.094=0.96$, and therefore, by (2), ${ }_{v-}^{v}=1 \cdot 15$, which differs only by 0.35 from the full slip. Thus the Selenites would obtain with a

* Such fantastically large condensations need not frighten us. They can be reduced if lioyle's law is replaced by some other appropriate form of relation between pressure and density. Boyle's law, which is by no means necessary, is here used only, as the simplest one, for the sake of illustration. 
Michelson-Morley experiment a pronounced positive effect. But enough has now been suid in illustration of the formulafor the condensation and for the slip.

5. Before passing to consider the Eclipse result it may be well to generalize the condensation formula (3) for the case in which Boyle's law is replaced by any relation between the pressure and the density of the xther. The corresponding generalization of the slip-formula (2), not required for our present purposes, may be postponed to a later opportunity.

Let the pressure $p$ be any function of the density $\rho$ alone, and let there be any distribution of gravitating masses. Introduce the function, familiar from hydrodynamics,

$$
\Phi=\Phi(\rho)=\int_{\rho}^{d p} \cdot \cdot \cdot \cdot \cdot \cdot
$$

Then, in the state of equilibrium, and with $d m$ written for any mass-element in astronomical units,

$$
\Phi=\int \frac{d m}{r}, \quad \cdot \quad \cdot \quad \cdot \quad \cdot .
$$

where $r$ is the distance of the contemplated point from $d m$, and the integral, representing the total gravitational potential, extends over all material bodies. $\Phi$ being a known function of $\rho$, formula (6) gives the required relation. It will be seen from the definition (5) that the dimensions. of $\Phi$ (work per unit mass of ether) are those of a squared velocity. In order to bring this into evidence, let us recall that

$$
\mathfrak{v}=\sqrt{\frac{d p}{d \rho}} \cdot \cdot \cdot \cdot \cdot \cdot \cdot
$$

is the velocity of propagation of longitudinal waves in any compressible non-viscous fluid *. This velocity is, in general, a function of $\rho$, and becomes a constant for the special case of Boyle's law, namely, our previous $1 / \sqrt{\alpha}$. Using (7) and writing, as before, $\frac{d \rho}{\rho}=d \log s$, we have

$$
\Phi=\int \mathfrak{v}^{2} \cdot d \log s, \quad . \quad . \quad .
$$

the required form. The integral is to be extended from

* This result, known as the formula of Laplace, holds also for the most characteristic kind of waves-to wit, for a wave of longitudinal discontinuity (Hugoniot, Hadamard), for which it follows directly, without integration, from the hydrodynamical equations of motion. See, for instance, my 'Vectorial Mechanics', p. 169. 
$s=1$ (or $\log s=0$ ) to the actual value of the condensation. Thus the condensation formula (6) becomes

$$
\int \mathfrak{v}^{2} \cdot d \log s=\Omega, \quad . \quad \cdot \quad . \quad .
$$

where $\Omega$ has been written for the total grayitational potential at the place under consideration. For constant $\mathfrak{v}$ (Boyle's law), and for a single spherical body, the previous formula (3) reappears.

It will be kept in mind that although the æther is assumed to behave in this way (say, like a gas) with respect to slow processes, it can still propagate rapid transversal ligbtdisturbances as it it were an elastic solid (like the famous cobbler's wax of Lord Kelvin) ; but it will be best to think of light as of electromagnetic disturbances. The normal velocity $c$ of propagating them is another property of the xther, independent of that which is represented by $\mathfrak{v}$, and subjected only to slight variations with condensation, as will appear presently. The ratio of $\mathfrak{v}$ to $:$ will be of importance, but as to the longitudinal waves themselves, they are of no physical interest for the present and, on the other hand, are not likely to become a nuisance. For it is not in our power to produce them to any relevant extent, and even if they are generated and maintained by some gigantic natural processes, their only effect would be to alter very slightly, bere and there, the normal velocity of light-propagation.

If we wish to form an idea of the numerical value of $\mathfrak{v}$, or at least of its upper limit, for the case of Boyle's law, say, it is enough to take the value of $\sigma$ given above for the Sun, and to remember that $M / c^{2}=1.5 \mathrm{~km}$., and, in round figures, $R=7 \cdot 10^{5} \mathrm{~km}$. Then the result will be $\frac{\mathfrak{v}}{c}=8 \cdot 2 \cdot 10^{-6}$, that is to say, $\mathfrak{v}$ equal to about $2 \cdot 5 \mathrm{~km}$. per second ${ }^{*}$. This is quoted by the way only. But the ratio of these two velocities will be seen to acquire a particular interest in connexion with the recent astronomical discovery.

6. Let $c$, as before, stand for the propagation velocity of light in uncondensed ather, i.e. in absence of, or far away from, gravitating masses, and let $c^{\prime}$ be the light velocity at a place where the æether has undergone a condensation $s$. The question is : How are we to correlate $c^{\prime}$ with $s$ ? In other words : On what are we to base the optical behaviour of the æether modified by a condensation? The only reasonable

* If so, then the condensational disturbances due to the Earth and other planets, whose velocities exceed $\mathfrak{v}$, will be confined to conical regions as in Mach's famous experiments. 
answer is: On exprerience. For, clearly, we cannot deduce it relation, which is essentially electro-mechanical, from mechanical principles alone, or "from electromagnetism alone. Nor can we imitate the usual dispersion theory (which makes use of both kinds of principles), for we are interested in those portions of the ærher in which there are no atoms and no electrons.

In short, as was announced in section 3 , let us write down the required relation by utilizing the observational result obtained by the Eclipse Expedition. In other words, let us see what that relation must be like in order to give the observed effect.

Now, if we disregard the small discrepancies (which may be either due to accidental errors or, perhaps, due to a superposed slight ordinary refraction), the observed total deflexions of the rays passing near the Sun are represented by Einstein's formula (quite apart from his theory)

$$
\Delta \theta=\frac{1}{r_{0}} \frac{4 M}{c^{2}}
$$

where $r_{0}$ is the minimum distance of the (undeflected) ray from the Sun's centre, and it can easily be shown that such will be the case ${ }^{*}$ if the refractive index $n=c / c^{\prime}$ at any distance $r>R$ from the Sun's centre be determined by

$$
n^{2}=1+\frac{4 M}{c^{2} r^{2}}
$$

or, denoting the potential by $\Omega$, and generalizing to any distribution of gravitational matter,

$$
n^{2}=1+\frac{4 \Omega}{c^{2}} \text {. . . . . . . }
$$

[This, in fact, is the formula which would follow at once from Einstein's approximate line-element

for a "static" field.]

$$
d s^{2}=c^{2} d t^{2}\left(1-\frac{2 \Omega}{c^{2}}\right)-\left(d x^{2}+d y^{2}+d c^{2}\right)\left(1+\frac{2 \Omega}{e^{2}}\right),
$$

In order to obtain the required relation, that is to say the assumption to be made "n the optical behaviour of the condensed rether, it is enough to combine equation (9) with our last equation (8), which gives

$$
n^{2}-1=\frac{4}{c^{2}} \int \mathfrak{v}^{2} \cdot d \log s . . . \quad . \quad .
$$

* Approximately, that is, for small $\Delta \theta$, and consequently for a refractive index but little differing from unity. 
Such, then, would be the required refractivity of the condensed rther, obeying any law $p=f^{\prime \prime}(\rho)$. In particular, if it obeys Boyle's law, we have

$$
n^{2}=1+4 \frac{\mathfrak{y}^{2}}{c^{2}} \cdot \log s, \quad . \quad . \quad . \quad .
$$

which is of a surprisingly simple form, and reads: $n^{2}-1$ equal to four times the logarithm of condensation multiplied $b_{y}$ the squared ratio of the two velocities of propagation characterizing the other.

Notwithstanding this temptingly simple form of the relation, I shall not try to "deduce" it from things more familiar. I prefer to regard it as an assumption, dictated by observation.

If the reader so desires, he can write $n^{2}-1=4 w / c^{2}$, where $w$ is the work, per unit mass of matter, done by the gravitational field in condeusing the rether. The small fraction $n^{2}-1$ being known from the Eclipse results (for any $r$ ), the numerical value of this work is determined without any further assumptions. If we agree to the lowest estimate of $\log s$ at the Sun's surface, as required by the aberration theory, we can also evaluate separately the ratio $\mathfrak{v} / c$, as already mentioned. This, howerer, is only a secondary matter.

7. Some. details and further implications of the StokesPlanck other theory, supplemented by assumption (10), must be postponed to a later opportunity. Here it will be enough to add only a few more general remarks. It will be kept in mind that the proposed theory would account not only for the observed astronomical aberration and for the older terrestrial optisal nil-effects, but manifestly also for the nileffect of the Michelson-Morley experiment. The hending of rays round the more massive celestial bodies would be only a by-product of the theory. Again, in view of the exceedingly small condensation of the xther round single atoms or corpuscles there will be no difficulty in working out a satisfactory electromagnetic theory of ponderable media. The proposed theory would also have the advantage of not predicting the obstinately absent gravitational shift of the spectrum lines. It might also react, in part at least, upon the 1905 relativity, depriving it of its indispensability in most cases, but by no means banishing it from the whole domain of physicomathematical investigations. Finally, the just objections raised by the advocates of the physical principle of causality against the fixed and homogeneous ather of Fresnel-Lorentz. would not apply to Stokes's modified æother. For this 
latter would by no ineans be a mere framework of yeference axes and, as such, illegitimately privileged. For in referring a class of phenomena to the æether here advocated we would ultimately refer them to assignable physical things, namely those most massive gigantic bodies which, so to speak, have the strongest grip upon that medium. It is, among other things, this latter remark that I hope to make particularly clear at an early opportunity.

London,

December 22, 1919.

XVI. Note on a Possible Structure for the Ether. By Sir Oliver Lodge*.

$D^{\mathrm{R}}$

R. SIIJBERSTEIN'S communication gives me an opportunity for calling attention to a paper of mine on many points in connexion with the ether which must surely be of interest even to those who are contemplating the abandonment of that medium. In that paper an estimate is made of etherial density, and an attempt to measure experimentally its lower limit is described; there are also comments of interest from Sir Joseph Larmor and Sir J. J. Thomson. The paper is in the Phil. Mag. ser. 6, vol. xiii. pp. 488-506, and is of date April 1907; though among other things it relates experiments conducted in and about 1893 .

The transmission of transverse vibrations like light shows that the ether cannot be a mere structureless fluid; and if it is to be treated dynamically, which at first is surely a legitimate attempt, it must have propertics akin to what we call, in matter, Rigidity and Inertia. Its inertia must be something fundamental, which underlies and accounts for the inertia we perceive in matter, possibly in a way having some analogy with a motion of a solid through a perfect fluid. For when an electric charge is moved, a magnetic field in the shape of an ether vortex-ring is generated (with an energy of circulation per unit volume equal to $\left.\mu(e u \sin \theta)^{2} / \delta \pi r^{4}\right)$, and this confers upon the charge its observed momentum if the modium has the requisite density (see Phil. Mag., April 1907, vol. xiii. p. 492). The rigidity may be explicable hydrodynamically by a vortex circulation-a turbulent motion having a circulatory velocity of the same order as that of the waves which the medium is able to transmit.

In Lord Kelvin's laminar vortex arrangement the velocity

* Communicated by the Author. 